\title{
Flow Simulation as a Support to Predict Shape of Plasma Beam Affected by the Nozzle Geometry
}

\author{
Miloslav Málek ${ }^{1, *}$, Miloš Mičian ${ }^{1}$, and Augustín Sládek ${ }^{1}$ \\ ${ }^{1}$ University of Žilina, Faculty of Mechanical Engineering, Univerzitná 1, 01026 Žilina, Slovakia
}

\begin{abstract}
This paper deals with flow simulation of plasma beam shape affected by the different nozzle geometry. The flow simulations for different nozzles geometry were made in simulation software AnsysFluent. The evaluation of flow simulations was based on comparing shapes of the flow media out from the modified nozzle orifice against reference nozzle. There were investigated 8 different modification of nozzle orifice. Modified nozzle n. 7 (in the shape of a Laval nozzle) has achieved significant improvement from all simulated. There were observed 3 cores of plasma beam, which could help blow dross out from cutting gap. Investigated results serve for further research.
\end{abstract}

\section{Introduction}

Plasma jet and arc processes are widely used for cutting, welding and coating of metals. The shielding gases as well as their flow profiles near the arc have a decisive influence on the machining results [1]. One of the modern technologies widely used is Plasma Arc Cutting (PAC) which is a far better metal cutting technique in some aspects when compared to conventional cutting techniques [2]. In this process, an inert gas (in some units, compressed air) is blown at high speed out of nozzle, at the same time an electrical arc is formed through that gas from the nozzle to the surface being cut, turning some of that gas to plasma [3]. Plasma is a jet or beam of ionized gas capable of conducting electricity. This ionized jet or beam produces extreme heat of around $33000{ }^{\circ} \mathrm{C}$ by recombination of ions and electrons into atoms and atoms into molecules. This heat is sufficiently high to melt and remove the metal [4 - 6]. Plasma looks and beahves like a gas but has distinctive difference, it conducts electrical charge [2]. The role of working fluid is to make the protection for the outer core of the PAC torch for the safe and protected operation. Hence the working fluid is very much in PAC and there are many fluid available and compatible for the PAC [7].

On of the biggest problems in plasma arc cutting is dross formatiuon on the worse side of cut. The amount of dross depends of lot parameters i.e. types of materials, cutting speed, currents, nozzle and flow rate [4]. The geometry of the nozzle causes significant increase in

\footnotetext{
* Corresponding author: miloslav.malek@,fstroj.uniza.sk
} 
inert gas speed and temperature [2]. The plasma is sufficiently hot to melt the metal being cut and moves sufficiently fast to blow molten metal away from the cut [3]. The gas pressure and current required to cut a work piece is dependent on various factors for example scanning speed, gas pressure and cutting height [2].

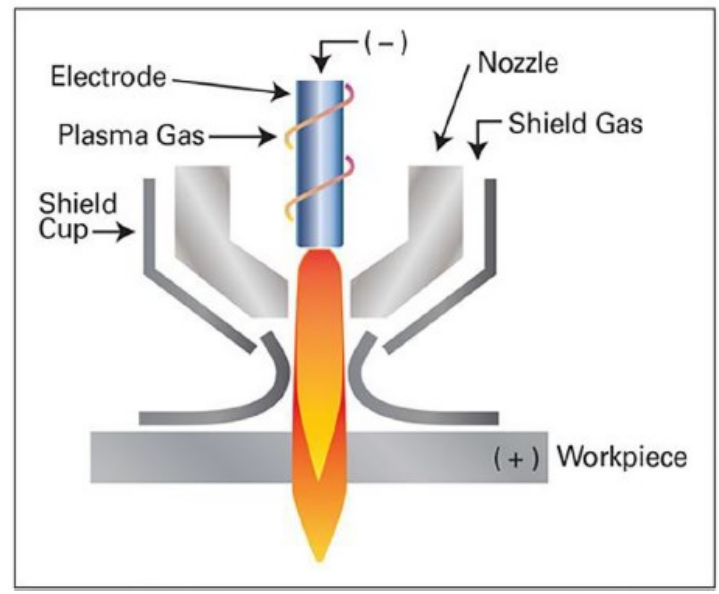

Fig. 1. Plasma arc cutting process [1].

A laval nozzle is a convergent-divergent nozzle that was invented by Gustav Patrik de Laval in 1888 for steam engine applications to produce a supersonic flow in the divergent section. The expansion state and internal flow line of the Laval nozzle are important parametrs that determine the structure of the air jet flow field as shown in Figure 2. The expansion state is characterized by the nozzle pressure ration $n$, i.e., the ratio of the nozzle outlet static pressure $P_{e}$ to atmospheric pressure. When $n<1$, it corresponds to an overexpanded jet, and $n=1$ correspond to an full-expansion jet. Additionally, $n>1$ corresponds to an underexpanded jet wherein $1<n<1.15$ corresponds to a low underexpanded jet and $n \geq 2$ corresponds to a highly underexpanded jet $[8,9]$.

Ansys Fluent is the industry-leading fluid simulation software used to predict fluid flow, heat and mass transfer, chemical reactions and other related phenomena. Known for delivering the most accurate solutions in the industry without compromise, Fluent's advanced physics modeling capabilities include cutting-edge turbulence models, multiphase flows, heat transfer, combustion, shape optimization, multiphysics and much more [10].

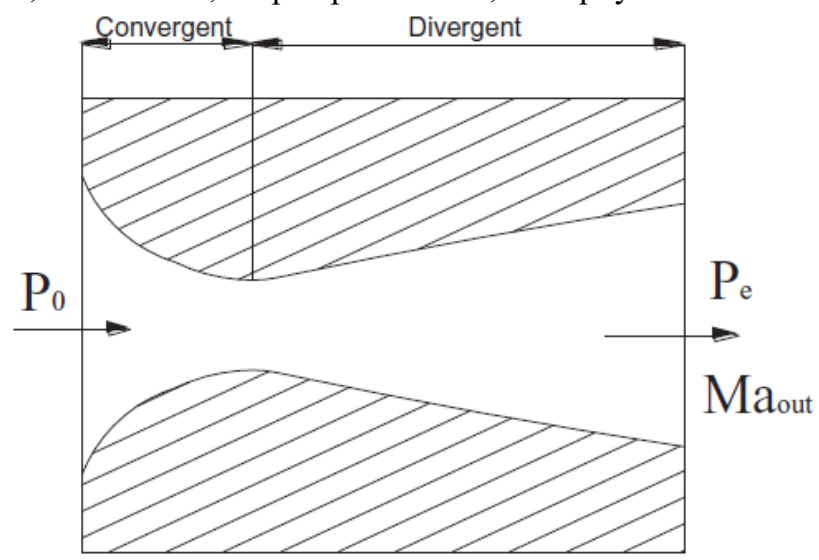

Fig. 2. Structure of the Laval nozzle [7]. 


\section{Preparation of simulated models}

First of all, plasma torch was measured and then 3D model was created. For simulations was used simplified model consist of nozzle and electrode, in 2D shape (Figure 3). The experiments consist of simulating a plasma flow using 8 different modified nozzle orifice shapes. Shape modification of the nozzle orifice kept these rules:

- The easiest manufactured way by conventional technologies,

- Low production/modification time,

- Low financial costs of manufactured.

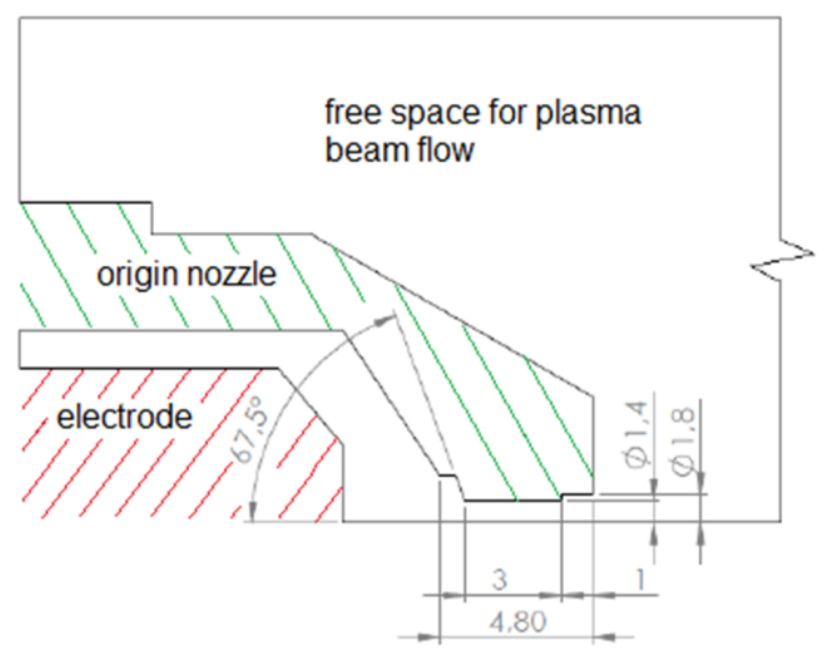

Fig. 3. 2D schematic model of nozzle with electrode for simulation a plasma flow.

For simplified and reduced computing time were used mentioned 2D models. Moreover, the task had symmetrical character, so the axis symmetry was placed identically with axis of nozzle and electrode, which means even more reduction in computing time.

Automatically generated net of finite elements was coarse and inaccurate. The modification of finite elements net consists of adding more finite elements, modify function "Skewness" and significant increasing finite elements in critical area. Sharp or significant dimensional changes and wall were marked as critical area (Figure 4). The "Bias" function was used to modify net around the wall. After appropriate adjustment of finite elements net was model prepared to define boundary conditions. Modified net has 33.8 times more finite elements compared to an automatically generated net, Figure 5. 


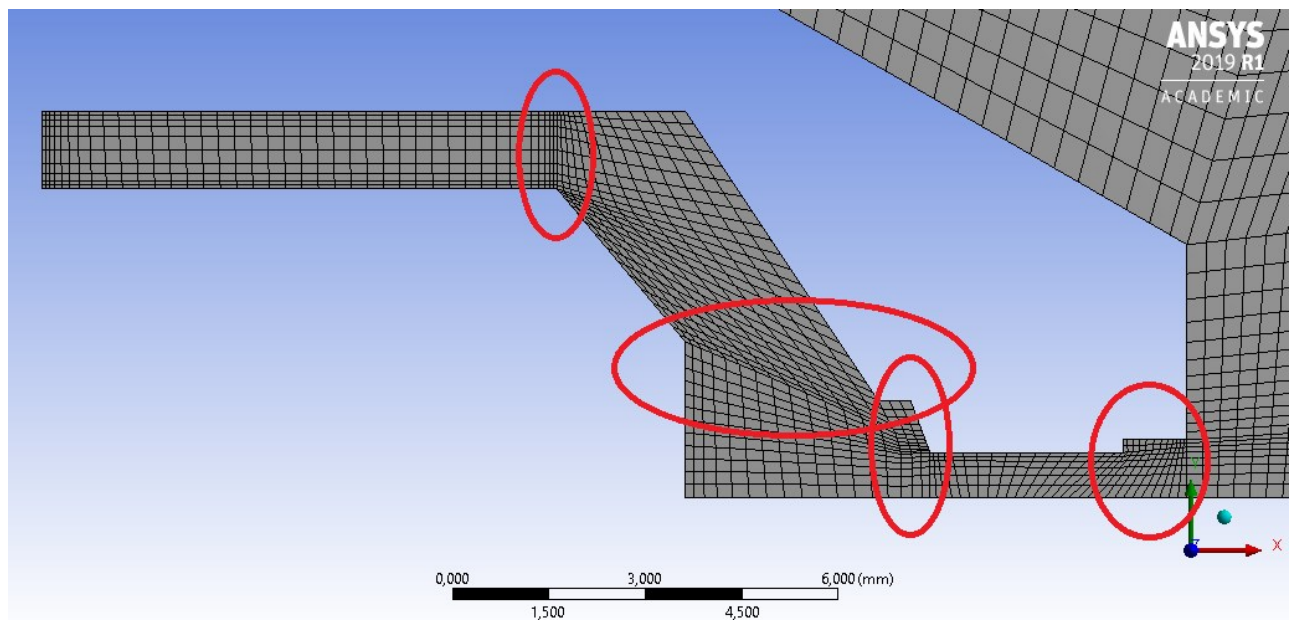

Fig. 4. Identification of critical areas on the model (circle marks).
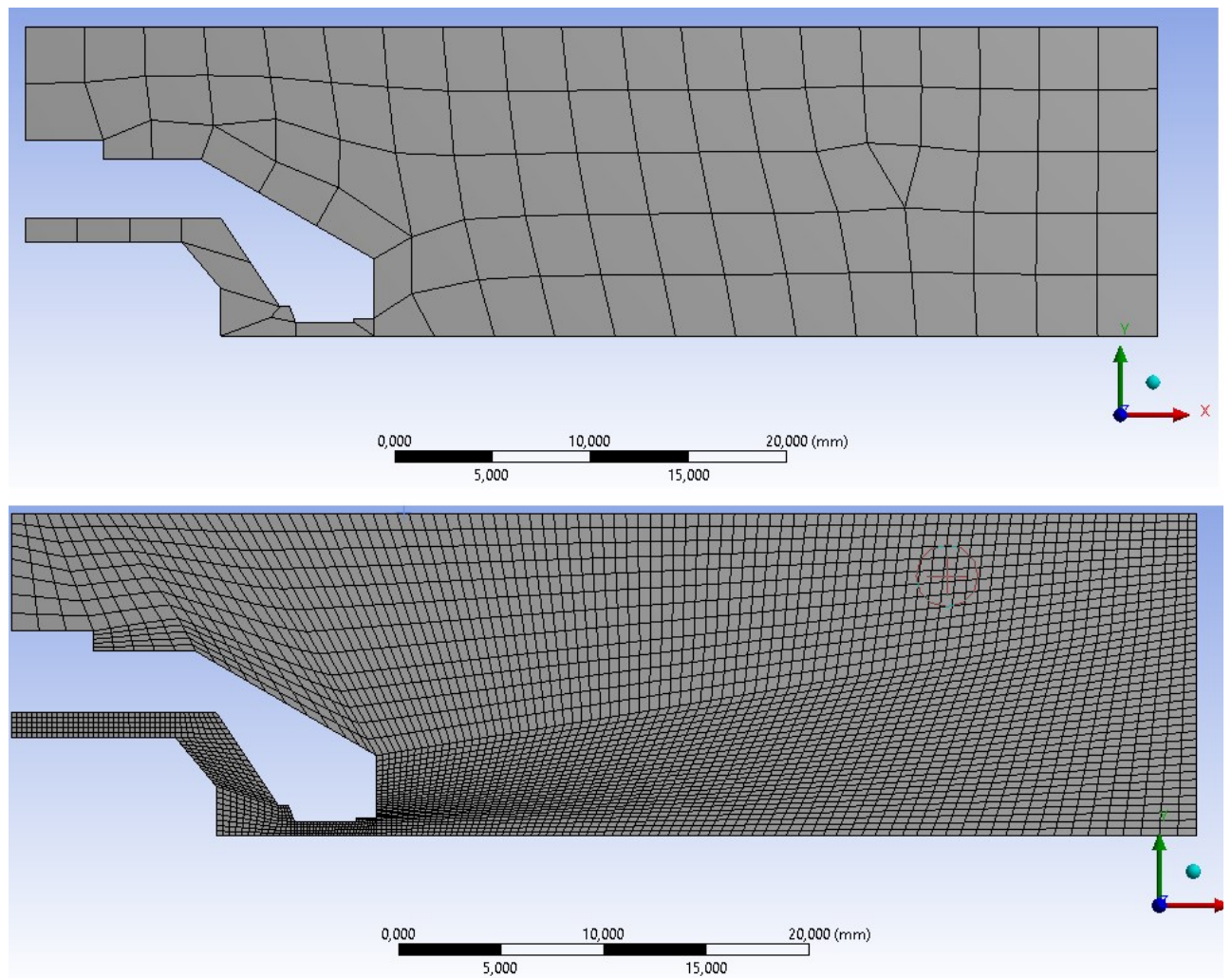

Fig. 5. Comparation automatically generated net with adjustement net.

The boundary condition, as wall, axis symmetry, inlet and outlet, were set up, Figure 6. Properties of plasma i.e. heat conductivity, density (electron density), effect of magnetic field, plasma beam frequency, viscosity (Newtonian or non-Newtonian fluid), plasma beam flow affected by electric arc and others is complex issue. In this research, the simulations were simplified as much as possible in order to obtain relevant results for further investigation. Inlet pressure and temperature were set up at $2.26 \mathrm{MPa}$ and $1200 \mathrm{~K}$. 
The outlet pressure and temperature were set up at $39.4 \mathrm{kPa}$ and $273.2 \mathrm{~K}$. The number of iteration was 4500 and fluid medium was ideal gas with Sutherland model viscosity.

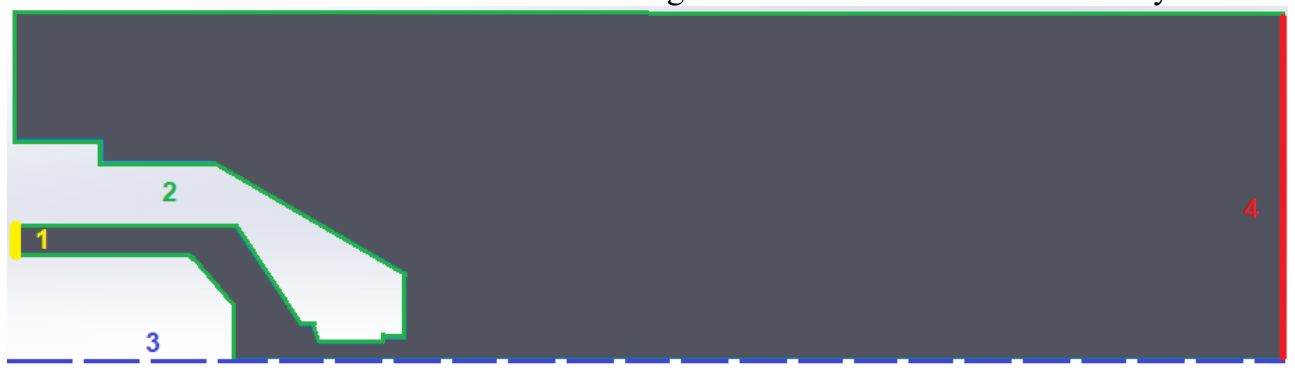

Fig. 6. Boundaries for the simulation of flow; 1 - flow input, 2 - impenetrable walls, 3 - axes of symmetry, 4 - flow output.

\section{Results}

Flow simulation is focused to achieve plasma beam shape flow out of modified nozzle orifice and then compared with origin nozzle shape.

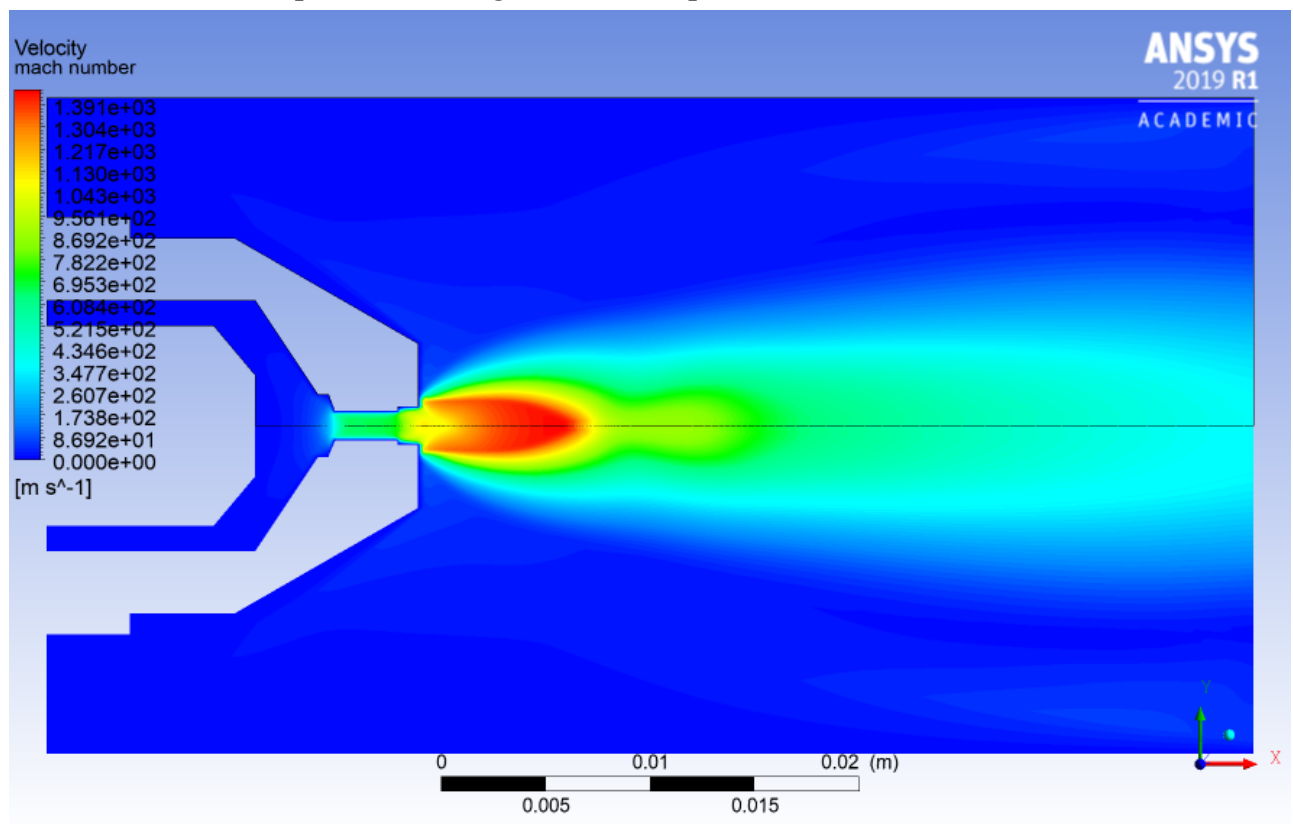

Fig. 7. Reference nozzle flow simulation.

Figure 7 shows results of flow simulation investigated on the origin nozzle shape (reference sample). Core formation of fluid medium represented by yellow outline was observed. All modified nozzle were simulated and compared with reference nozzle. Every modification of nozzle orifice kept the rules mentioned above expect last two modifications. The Figure 8 shows all simulated results compare to the reference nozzle (Figure 8-a to Figure 8-i upper half of picture). 


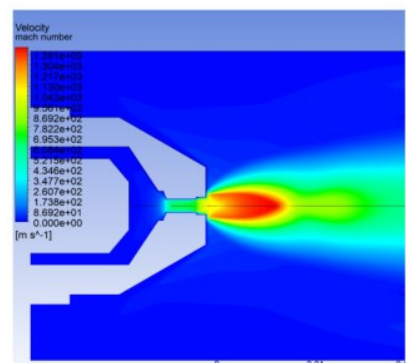

a)

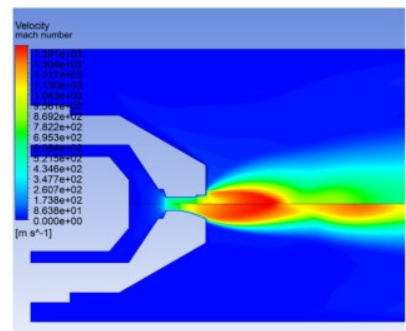

d) $-1-$

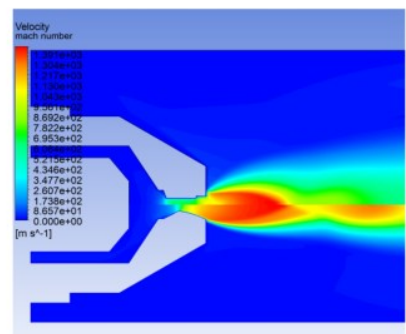

g)

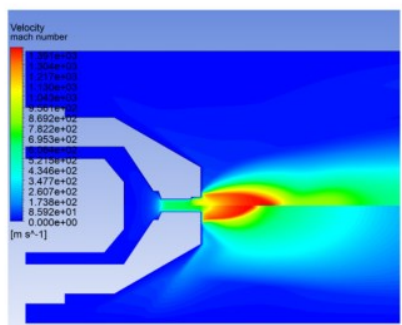

b) $-\ldots$

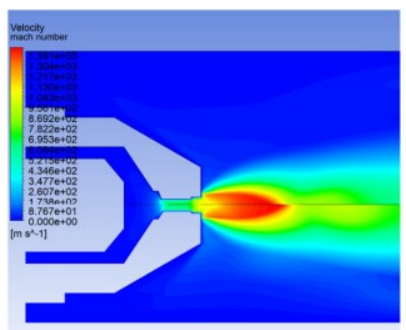

e) - -

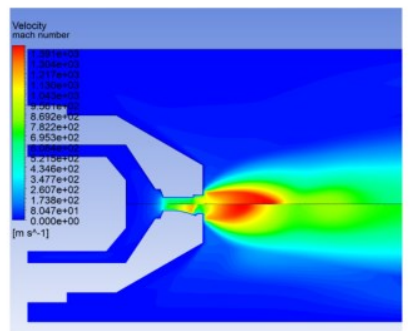

h)
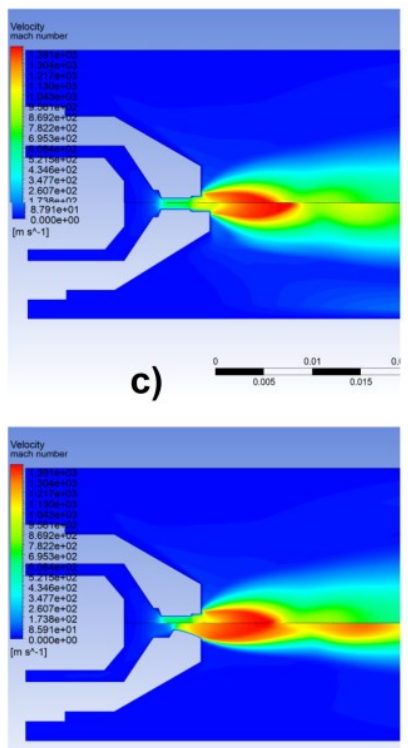

f) $-\ldots$

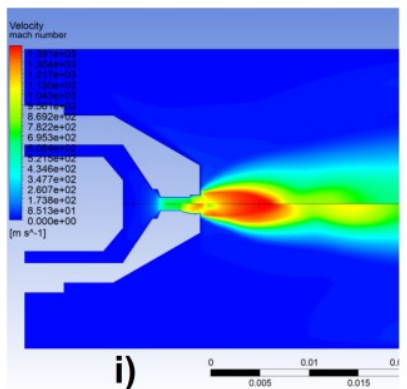

Fig. 8. Results of comparison of flow simulation; a) - reference nozzle; b) to i) modified nozzles (upper hlaf of picture is reference nozzle and lower half is modified nozzle).

From the results of the simulation calculations and based on a comparison with the plasma beam flow in the reference nozzle, the most suitable shape for the cutting process is the modified shape marked as No. 7 (Figure 8-f). The plasma beam shape for this modification had significant different shape against reference nozzle. The core of the plasma beam (yellow outline) had longer range, moreover there was observed second and third core area, which could help to blow dross out from cutting gap (Figure 9). This modification fulfils manufacture requirements. 


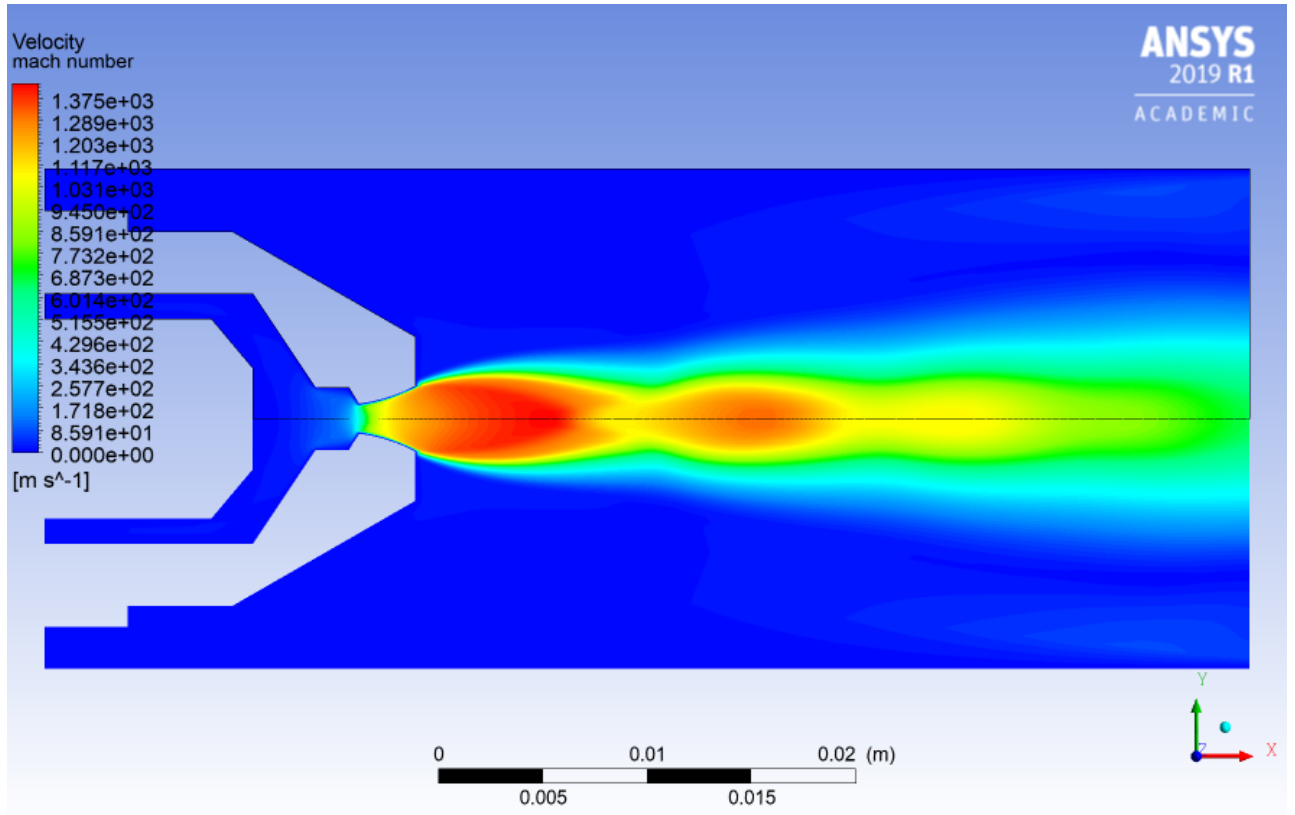

Fig. 9. Modificated nozzle No. 7 flow simulation.

\section{Conclusion}

Flow simulation of gas fluid through nozzle with electrode affected by different nozzle orifice modifications was investigated in this paper. This research served to further investigation, to predict suitable design for real experiments. Modified nozzle No. 7 indicated the best results from all 8 modified shape of nozzle orifice. This modification fulfilled requirements as easy manufacture by conventional technology, low production time and costs, and moreover it is an easily repeatable process. The simulation confirmed the prediction that Laval nozzle shape is suitable for supersonic flow which is an accompanying phenomenon of every plasma arc cutting.

This research was funded by KEGA, grant number KEGA 009ŽU-4/2019; and VEGA, grant number VEGA $1 / 0951 / 17$. The authors hereby thank these agencies for their support.

\section{References}

1. M. Schnick, U. Füssel, J. Zschetzsche, Mod. For Mat. Proc. (2006)

2. A. Rajeshkannan, M. Ali, R. Prakash, A. K. Jeevanantham, K. Jayaram, Mat. Tod. 24 1122-1131, (2020)

3. A. Moarrefeyadeh, WSEAS Transac. on Appl. and Theor. Mech. 6 (2011)

4. D. N. Sharma, J. R. Kumar, Mat. Tod. (2020)

5. A. Suresh, G. Diwakar, Mat. Tod. (2020)

6. Y. Liu, J. Zhang, J. Wei, X. Liu, Powd. Techn. 364, 343-362 (2020)

7. https://www.ansys.com/products/fluids/ansys-fluent

8. M. Mičian, D. Harmaniak, F. Nový, J. Winczek, J. Moravec, L. Trško, Metals, 10 (2), $229,(2020)$ 
9. J. Lago, L. Trško, M. Jambor, F. Nový, O. Bokůvka, M. Mičian, F. Pastorek, Metals 9 (6), 619, (2019)

10. M. Bruna, L. Kucharcik, Materiali in Tehnologije, 48 (6), 949-952 (2014) 